\title{
Spontaneous Symmetry Breakdown in the Abelian Higgs Model
}

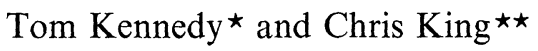 \\ Department of Physics, Princeton University, Princeton, NJ 08544, USA
}

\begin{abstract}
For the abelian Higgs model we introduce a new gauge invariant observable which in Landau gauge is $\phi(x) \bar{\phi}(y)$. In three or more dimensions this observable is used to show that the global gauge symmetry is spontaneously broken in the lattice theory for a suitable range of parameters. This observable also provides a gauge invariant order parameter for the phase transition in this model.
\end{abstract}

\section{Introduction}

The Higgs mechanism plays an important role in determining the particle structure of gauge field theories. This mechanism provides a natural way for the gauge bosons to acquire a mass. The standard explanation of this mass generation assumes that the global gauge symmetry is spontaneously broken [2].

One of the simplest gauge theories which is believed to exhibit spontaneous symmetry breaking and the Higgs mechanism is the abelian Higgs model. It has been rigorously established in Euclidean lattice versions of this model that there exists a phase in which the photon is massless and a phase in which it is massive $[1,7,8,17,18]$.

These proofs of the Higgs mechanism are quite different from the heuristic explanation and do not address the question of spontaneous symmetry breaking. In this paper we introduce a new order parameter and use it to prove spontaneous symmetry breaking in the abelian Higgs model in dimension three or more. Our result is proven for the Euclidean lattice version of the model with a noncompact action.

In the abelian Higgs model spontaneous symmetry breakdown would mean that $\langle\phi(x)\rangle=\phi_{0} \neq 0$, where $\phi(x)$ is the Higgs field. This would mean that the Higgs field has long range order (LRO) like the spins in a ferromagnet at low temperature. However, $\phi(x)$ is not a gauge invariant observable, so this picture

* Research supported by U.S. National Science Foundation grant PHY8116101-A03

$\star \star$ Research supported by U.S. National Science Foundation grant PHY8117463 
may be true in some gauges and false in others. For example, Fröhlich et al. [10] have shown it is false in axial gauge for the lattice theory.

By analogy with the ferromagnet, another criterion for LRO is that the twopoint function $\langle\phi(x) \bar{\phi}(y)\rangle$ does not decay to zero as $|x-y| \rightarrow \infty$. A natural gauge invariant observable that might be used to test this idea is the string $\phi(x) \exp \left(-i e \int_{x}^{y} \mathbf{A} \cdot d \mathbf{l}\right) \bar{\phi}(y)$. However, the vacuum expectation value of this observable always decays to zero for the lattice theory [10]. Other criteria for LRO based on the behavior of this observable have been introduced in $[3,13]$.

Another gauge invariant observable involving $\phi(x) \bar{\phi}(y)$ is the smeared string

$$
G(x, y)=\phi(x) \exp \left[-i e \int d z \mathbf{A}(z) \cdot \mathbf{h}(z)\right] \bar{\phi}(y),
$$

where $\mathbf{h}$ is the $d$-dimensional Coulomb field generated by charges -1 at $x$ and +1 at $y$. We are working with the Euclidean theory, so $\mathbf{A}$ and $\mathbf{h}$ are $d$-component vectors, and $z$ is integrated over $\mathbb{R}^{d}$. Under a gauge transformation $\mathbf{A} \rightarrow \mathbf{A}+\frac{1}{e} \nabla \chi$, $\phi \rightarrow e^{i \chi} \phi$, the smeared string transforms as

$$
i e \int d z \mathbf{A}(z) \cdot \mathbf{h}(z) \rightarrow i e \int d z \mathbf{A}(z) \cdot \mathbf{h}(z)+i \int d z \nabla \chi(z) \cdot \mathbf{h}(z) .
$$

After integrating by parts and using $\boldsymbol{\nabla} \cdot \mathbf{h}(z)=-\delta(z-x)+\delta(z-y)$, the second term becomes $i \chi(x)-i \chi(y)$. This exactly compensates for the effect of the gauge transformation on $\phi(x) \bar{\phi}(y)$, so $G(x, y)$ is gauge invariant. In Landau gauge $(\boldsymbol{\nabla} \cdot \mathbf{A}=0), G(x, y)$ is equal to $\phi(x) \bar{\phi}(y)$, since $\mathbf{h}$ is the gradient of a potential.

The expectation that we use is defined with a non-compact gauge field $\mathbf{A}(x)$ and a gauge-fixing term $\frac{1}{2 \alpha} \sum_{z}|\boldsymbol{\nabla} \cdot \mathbf{A}(z)|^{2}$ in the action. In three or more dimensions this model has been shown to have a phase in which the photon is massive [1] and a phase in which it is massless [8]. We will refer to these phases as the Higgs phase and QED phase, respectively.

Our main result is that for the lattice theory in three or more dimensions there is a range of parameters for which $\langle G(x, y)\rangle$ does not decay to zero as $|x-y| \rightarrow \infty$. This range of parameters overlaps the Higgs phase. We also prove that $\langle G(x, y)\rangle$ decays to zero for a range of parameters overlapping the QED phase. Thus we prove that the model has a phase transition with order parameter

$$
G_{\infty}=\lim _{|x-y| \rightarrow \infty}\langle G(x, y)\rangle .
$$

These results were announced in [15]. We do not prove that this transition coincides with the transition between the Higgs and QED phases.

The expectation $\langle G(x, y)\rangle$ is independent of $\alpha$, since $G(x, y)$ is gauge invariant. Landau gauge is obtained by taking $\alpha=0$, so

$$
\langle G(x, y)\rangle=\langle\phi(x) \bar{\phi}(y)\rangle_{\text {Landau }} .
$$

Therefore we have shown that the Higgs field has LRO in Landau gauge in the same sense as the spins in the classical, $d$-dimensional $X-Y$ model. We conjecture that the translation invariant pure phases of the lattice abelian Higgs model in 
three or more dimensions are parameterized by an angle $\theta \in[0,2 \pi)$, and that

$$
\langle\phi(x)\rangle_{\text {Landau }}=c e^{i \theta},
$$

in the pure phase labelled by $\theta$. We do not prove this conjecture here. Our definition of the expectation does not break the global U(1) symmetry. So this produces a mixed phase with $\langle\phi(x)\rangle_{\text {Landau }}=0$.

The existence of LRO in Landau gauge does not mean that $\phi(x)$ has LRO in other gauges. For example, we can consider the decay of $\langle\phi(x) \bar{\phi}(y)\rangle$ when the parameter $\alpha$ in the gauge-fixing term is not zero. We prove that in this case $\langle\phi(x) \bar{\phi}(y)\rangle$ decays to zero as $|x-y| \rightarrow \infty$ in dimension four or less.

Our last result concerns the decay of the truncated two-point function. For a region of parameters where $G_{\infty}$ is not zero, we prove that $\langle G(x, y)\rangle-G_{\infty}$ decays to zero as $|x-y|^{2-d}$. This massless behavior indicates the presence of Goldstone bosons. However, we do not expect these massless particles to couple to states in the physical Hilbert space (see [2]).

Our proofs rely on the transformation of Bałaban et al. [1] to write our noncompact model in a compact formulation. In the non-compact model there is a global U(1) symmetry, so a Peierls argument or standard expansion methods cannot be used to prove LRO. This continuous symmetry is integrated out by the transformation of [1], and so does not appear in the compact formulation. Thus we can use a standard polymer expansion for the compact model. Proofs of phase transitions in other lattice models with a continuous symmetry which use a transformation to remove the global continuous symmetry include $[14,12]$.

It would be interesting to extend our result to the compact version of the $U(1)$ Higgs model. It would also be of interest to prove a corresponding result for the non-abelian Higgs models. However, it is difficult to see how the smeared string can be constructed for these theories. It might be possible to work directly in Landau gauge and to prove that $\langle\phi(x)\rangle_{\text {Landau }}$ is also an order parameter for these theories.

We define our model and state the results in Sect. 2. Some of the easier results are proven in this section. Section three contains the proof of long range order. Massless decay of the truncated two point function is established in Sect. 4. Section five and Appendix B are devoted to technical estimates. A correlation inequality relating the fixed and variable length models is proven in Appendix A.

\section{Statement of Results}

Our model is defined on a finite, $d$-dimensional rectangular lattice $\Lambda$ with unit spacing. We will prove the existence of the infinite volume limit in Sect. 4. We denote by $\Lambda^{*}$ and $\Lambda^{* *}$ the oriented bonds and plaquettes respectively in $\Lambda$. 0 -forms, 1 -forms, and 2 -forms are functions on $\Lambda, \Lambda^{*}$, and $\Lambda^{* *}$ respectively. The scalar field (or Higgs field) is a complex-valued 0 -form, which we write as $\phi(x)$ $=r(x) \exp [i \theta(x)]$, with $r(x) \in[0, \infty)$ and $\theta(x) \in[0,2 \pi)$. The vector field (or gauge field) is a real-valued 1 -form, written $A(b)$, where $b$ is a bond in $A^{*}$. We shall write $(f, g)$ for the $\ell^{2}$-inner product of $p$-forms. Thus if $f, g$ are 1 -forms, $(f, g)$ involves a sum over $\Lambda^{*}$; if 2-forms a sum over $\Lambda^{* *}$. The lattice exterior derivative on $\Lambda$ is $d$, and its adjoint with respect to $($,$) is d^{*}$ (for a review of notation see $[9,14]$ ). 
We have results for a fixed length scalar field $(|\phi(x)|=a)$ and for the more general case of a variable length field. The action of the fixed length model is

$$
S(A, \theta)=\frac{1}{2}(d A, d A)+\frac{1}{2 \alpha}\left(d^{*} A, d^{*} A\right)-a^{2} \sum_{b \in \Lambda^{*}} \cos [d \theta(b)-e A(b)] .
$$

The term $\frac{1}{2 \alpha}\left(d^{*} A, d^{*} A\right)$ is the standard gauge-fixing term; $\alpha=1$ corresponds to Feynman gauge and $\alpha=0$ to Landau gauge. For the variable length scalar field we use the action

$$
S^{\prime}(A, \phi)=\frac{1}{2}(d A, d A)+\frac{1}{2 \alpha}\left(d^{*} A, d^{*} A\right)+\frac{1}{2} \sum_{b \in \Lambda^{*}}|D \phi(b)|^{2}+\sum_{x \in \Lambda} V(\phi(x)),
$$

where the covariant derivative is

$$
D \phi(b)=\phi\left(b_{+}\right)-\exp [i e A(b)] \phi\left(b_{-}\right),
$$

and $b_{+}, b_{-}$are the endpoints of $b$. The single site potential is $V(\phi(x))=\frac{1}{2} m^{2}|\phi(x)|^{2}$ $+\lambda|\phi(x)|^{4}$, where $\lambda>0$ and $m^{2}$ can be positive or negative. The potential can be a single or a double well depending on the sign of $m^{2}$. By taking $m^{2}=-4 \lambda a^{2}$ and sending $\lambda$ to infinity, the variable length model with action (2.2) reduces to the fixed length model with action (2.1).

For any functional $F$ defined on field configurations on $\Lambda$, we introduce expectations by

$$
\begin{aligned}
& \langle F\rangle=Z^{-1} \int D A \int D \theta F \exp [-S(A, \theta)], \\
& \langle F\rangle^{\prime}=Z^{\prime-1} \int D A \int D \phi F \exp \left[-S^{\prime}(A, \phi)\right],
\end{aligned}
$$

where $Z, Z^{\prime}$ are partition functions that normalise the expectations. We will use the non-compact version of the model in this paper, so

$$
\int D A=\prod_{b \in \Lambda^{*}} \int_{-\infty}^{\infty} d A(b) .
$$

Also $D \theta$ and $D \phi$ denote products of Lebesgue measure on the circle and on $\mathbb{C}$. For a gauge-invariant functional $F$, both $\langle F\rangle$ and $\langle F\rangle^{\prime}$ are independent of $\alpha$, the parameter in the gauge-fixing term [6].

The inclusion of the gauge-fixing term $\frac{1}{2 \alpha}\left(d^{*} A, d^{*} A\right)$ and our choice of boundary conditions make the integrals in (2.4) converge. In other words, the equations $d A=0$ and $d^{*} A=0$ have the unique solution $A=0$ (recall that $d^{*}$ is the adjoint of $d$ restricted to $\Lambda$, so it does not annihilate constants). However for a 0 -form $f$, the equation $d f=0$ is satisfied by $f=$ const. So the Laplacian on 0 -forms $\Delta=d^{*} d$ is only invertible on the orthogonal complement of the constant functions, and we denote its inverse on this subspace by $C$.

We can now introduce the smeared string observable described in the introduction. Define

$$
G(x, y)=\phi(x) e^{-i e(A, h)} \bar{\phi}(y),
$$

where $h$ is given by

$$
h=d C g,
$$


and $g=\delta_{x}-\delta_{y}$. We write $\delta_{x}$ for the lattice delta function at site $x$. So $h$ is the electric field produced by charges -1 at $x$ and +1 at $y$. In $d$ dimensions, the electric field produced by a unit charge at the origin decays as $r^{1-d}$, which is square integrable for $d \geqq 3$. It will be crucial for our results that $h$ is $\ell^{2}$ uniformly in $x, y$ and $A$ for $d \geqq 3$.

We can rewrite $(2.5)$ as

$$
G(x, y)=r(x) r(y) e^{i(\theta, g)-i e(A, h)} .
$$
Under a gauge transformation $\theta \mapsto \theta+\chi, A \mapsto A+\frac{1}{e} d \chi$, the term $e(A, h)$ transforms
as

$$
e(A, h) \mapsto e(A, h)+(d \chi, h) .
$$

The gauge invariance of $G(x, y)$ follows from the fact that $d^{*} h=g$, since this gives $(d \chi, h)=(\chi, g)$. Furthermore, since

$$
(A, h)=\left(d^{*} A, C g\right),
$$

it follows that in Landau gauge $\left(d^{*} A=0\right)$ the observable $G(x, y)$ reduces to $\phi(x) \bar{\phi}(y)$.

Our main result concerns $\langle G(x, y)\rangle$. The theorem below says that if $a$ is large and $e$ is small, then the smeared string has LRO, but if $a$ is small it does not. The main part of the proof is given in Sect. 3 .

Theorem 2.1. (i) For $d \geqq 3$ and for any $0<\gamma<1$, there are constants $e(\gamma), a(\gamma)$ such that for $e<e(\gamma)$ and $a>a(\gamma)$

$$
\langle G(x, y)\rangle \geqq \gamma a^{2} \forall x, y \in \Lambda .
$$

(ii) There are constants $\delta, \varrho, c$ such that for $a \leqq \delta$ and for all $e$,

$$
\langle G(x, y)\rangle \leqq c \exp [-\varrho|x-y|] \text {. }
$$

Remark. Theorem 2.1 proves that the model has a phase transition in dimension $d \geqq 3$. The natural order parameter for this transition is

$$
G_{\infty}=\lim _{|x-y| \rightarrow \infty}\langle G(x, y)\rangle .
$$

Proof of Theorem 2.1. The body of the proof of (i) appears in Sect. 3. We complete that proof here and also prove (ii). The invariance of the measure under $A \mapsto-A$, $\theta \mapsto-\theta$ implies that

$$
\langle G(x, y)\rangle=\langle r(x) r(y) \cos [(\theta, g)-e(A, h)]\rangle .
$$

To prove (ii), we use the correlation inequalities of Brydges et al. [6]. Recall that they prove the following result for all values of the parameters:

$$
\langle P(r) \cos [(n, \theta)+e(f, A)] ; Q(r) \cos [(m, \theta)+e(g, A)]\rangle^{\prime} \geqq 0,
$$

where $P, Q$ are polynomials in the variables $\{r(x)\}$ with positive coefficients, $n$ and $m$ are integer-valued 0 -forms and $f$ and $g$ are real-valued 1-forms. From (2.9) we immediately deduce that

$$
\frac{d}{d \lambda}\langle G(x, y)\rangle^{\prime} \leqq 0
$$



We will use this result later. Also by rescaling $A \mapsto \frac{1}{e} A$ and using standard
substitutions like

$$
\frac{1}{2}|d A(p)|^{2}=\lim _{\varepsilon \rightarrow 0} \varepsilon^{-2}[1-\cos (\varepsilon d A(p))],
$$

we get

$$
\frac{d}{d e}\langle G(x, y)\rangle^{\prime} \leqq 0
$$

Since (2.11) holds also for the fixed length case, we may bound $\langle G(x, y)\rangle$ from above by the same expectation with $e=0$. However this is exactly the spin-spin correlation of the $d$-dimensional $X-Y$ model at inverse temperature $\beta=a^{2}$. Therefore for $a$ sufficiently small, this decays exponentially.

We prove (i) in Sect. 3 for the range of parameters $e<e(\gamma)$, ae $>\mu(\gamma)$. Using (2.11), the result extends to the range $e<e(\gamma), a>a(\gamma)$ with $a(\gamma)=\mu(\gamma) / e(\gamma)$.

We point out that since taking $e=0$ gives the $X-Y$ model, Theorem 2.1 provides a new proof of LRO for the $X-Y$ model in $d \geqq 3$; for earlier proofs see $[11,12]$.

Corollary 2.2. In $d \geqq 3$, for sufficiently low temperature the two-point function of the classical $X-Y$ model is bounded away from zero.

As mentioned before, we can also show that the variable length model has a phase transition. If the Higgs potential is a deep double well, there is LRO for the smeared string. There is no LRO for a single well potential.

Theorem 2.3. (i) In $d \geqq 3$, for any $\lambda>0$ there are constants $e_{0}, R(\lambda)>0$ such that for all $e<e_{0}$ and $-m^{2}>R(\lambda)$,

$$
\langle G(x, y)\rangle^{\prime} \geqq c>0 \quad \forall x, y \in \Lambda .
$$

(ii) For $m^{2}>0$ and all $\lambda, e, d$,

$$
\langle G(x, y)\rangle^{\prime} \leqq c^{\prime} \exp [-m|x-y|],
$$

where $c^{\prime}$ depends only on $m$ and $d$.

Proof. Part (i) is proved in Appendix A, using a correlation inequality to relate $\langle G(x, y)\rangle$ and $\langle G(x, y)\rangle^{\prime}$. To prove (ii) we use (2.10) and (2.11) to bound $\langle G(x, y)\rangle$ from above by the two-point function of a free scalar field with mass $m$, from which (2.13) follows.

Theorem 2.1 shows that in Landau gauge the expectation of the observable $\phi(x) \bar{\phi}(y)$ is bounded away from zero for a suitable range of parameters in $d \geqq 3$, indicating that $\phi(x)$ has LRO. As an interesting contrast, we show that $\phi(x)$ does not have LRO for any choice of $\alpha$ different from zero. The proof uses a complex translation method similar to that in [16]. Although our theorem concerns the fixed length case, it is straightforward to extend it to the variable length model.

Theorem 2.4. For $d=3,4, \alpha \neq 0, e \neq 0$, and any $a>0$,

$$
\langle\phi(x) \bar{\phi}(y)\rangle \rightarrow 0 \quad \text { as } \quad|x-y| \rightarrow \infty .
$$


Proof. We will rewrite the action (2.1) as

$$
S(A, \theta, \beta)=\frac{1}{2}(d A, d A)+\frac{1}{2 \alpha}\left(d^{*} A, d^{*} A\right)-\beta \sum_{b \in \Lambda^{*}} \cos [d \theta(b)-e A(b)],
$$

and denote by $\langle\cdot\rangle(\beta)$ and $Z(\beta)$ the corresponding expectation and partition function. Then the correlation inequality (2.9) implies that

Therefore

$$
\frac{d}{d \beta}\left\langle e^{i(\theta, g)}\right\rangle(\beta) \geqq 0 \text {. }
$$

$$
\begin{aligned}
\langle\phi(x) \bar{\phi}(y)\rangle= & a^{2}\left\langle e^{i(\theta, g)}\right\rangle\left(a^{2}\right) \\
\leqq & a^{2}\left\langle e^{i(\theta, g)}\right\rangle(\infty) \\
= & a^{2} Z(\infty)^{-1} \int D A \int D \theta \exp \left[-\frac{1}{2}(d A, d A)-\frac{1}{2 \alpha}\left(d^{*} A, d^{*} A\right)\right] \\
& \cdot \prod_{b \in \Lambda^{*}}\left\{\sum_{n(b)} \delta(d \theta(b)-e A(b)+2 \pi n(b))\right\} \exp [i(\theta, g)] \\
= & a^{2} Z(\infty)^{-1} \int D \theta \sum_{n} \exp [i(\theta, g)] \\
& \cdot \exp \left\{-\frac{1}{2 e^{2}}\left(d \theta+2 \pi n,\left[d^{*} d+\frac{1}{\alpha} d d^{*}\right][d \theta+2 \pi n]\right)\right\},
\end{aligned}
$$

where the sum is over integer-valued 1-forms. Since the integrand in (2.15) is periodic in each $\theta(x)$ (the sum over $n$ is part of the integrand), we can make a complex translation in $\theta$. Define $H=\left(d^{*} d d^{*} d\right)^{-1}$ on the orthogonal complement of the constants, and translate $\theta$ in (2.15) by

$$
\theta \mapsto \theta+i e^{2} \alpha H g .
$$

Then after bounding the resulting complex integrand, we get

$$
|\langle\phi(x) \bar{\phi}(y)\rangle| \leqq a^{2} \exp \left[-\frac{1}{2} e^{2} \alpha(g, H g)\right] .
$$

As $|x-y| \rightarrow \infty,(g, H g)$ behaves asympiotically like $|x-y|$ in $d=3$ and like $\ln |x-y|$ in $d=4$, so this proves (2.14) for $\alpha e^{2}>0$.

Finally we state a result concerning the existence of massless excitations in the fixed length model. Recall that $G_{\infty}$ was defined as the limit of $\langle G(x, y)\rangle$ as $|x-y| \rightarrow \infty$.

Theorem 2.5. In $d \geqq 3$ there are constants $e_{0}$ and $\mu_{0}$ such that for $e<e_{0}$ and ae $>\mu_{0}$,

$$
\langle G(x, y)\rangle-G_{\infty}=\frac{\sigma G_{\infty}}{|x-y|^{d-2}}+O\left(\frac{1}{|x-y|^{d-1}}\right),
$$

where $\sigma$ is a positive constant.

Theorem 2.5 is proved in Sect. 4. At first sight it is surprising, since it implies the presence of Goldstone bosons in our theory, at least in Landau gauge. However it has been known for a long time [2] that when this model is quantized in Lorentz 
gauge (the continuation to Minkowski space of Landau gauge) Goldstone's theorem cannot be evaded. The Hilbert space contains Goldstone bosons, but these are orthogonal to the sector containing physical states. Although we do not prove it, presumably the Goldstone bosons of Theorem 2.5 do not couple to physical states.

\section{Proof of LRO}

We begin this section by applying the transformation of Bałaban et al. [1] which changes the non-compact model into a compact model. Then we perform a polymer expansion for this compact model. We use this expansion and some technical estimates to prove Theorem 2.1 (i). These estimates are proven in Sect. 5.

In [1] the transformation from a non-compact model to a compact model was applied to observables which were both gauge invariant and periodic in each $A(b)$. Our observable is not periodic, but the transformation can still be applied. As remarked in [1] the transformed observable will be a function of the vortices $v$ as well as the compact field $A$.

The result of the transformation for the un-normalized expectation of $G(x, y)$ is

$$
\begin{aligned}
& \int D A \int D \theta \exp [-S(A, \theta)] G(x, y)=N \sum_{v: d v=0} \int_{c} D A \\
& \cdot \exp \left[-\frac{1}{2}\left(d A+\frac{2 \pi}{e} v, d A+\frac{2 \pi}{e} v\right)+a^{2} \sum_{b} \cos (e A(b))\right] \\
& \cdot a^{2} \exp \left[-i e(A, h)-2 \pi i\left(h, n_{v}\right)\right] .
\end{aligned}
$$

The integration in the right-hand side is over $A(b) \in(-\pi / e, \pi / e)$. We have emphasized this with the subscript $c$ on the integral. The sum over $v$ is over integervalued, closed $(d v=0) 2$-forms. For each such 2-form $v, n_{v}$ is an integer valued 1 -form such that $d n_{v}=v$. $N$ is a constant which depends only on $\alpha$ and $e$.

The choice of the $n_{v}$ 's is not unique. At first glance, $\exp \left[-2 \pi i\left(h, n_{v}\right)\right]$ depends on this choice. However, it actually only depends on $v$. To see this let $n_{v}$ and $\bar{n}_{v}$ be 1 -forms with $d n_{v}=d \bar{n}_{v}=v$. Then there is an integer valued 0 -form $s$ such that $\bar{n}_{v}=n_{v}+d s$. Now $(h, d s)=\left(d^{*} h, s\right)=(g, s)$, which is an integer. So $\exp \left[-2 \pi i\left(h, n_{v}\right)\right]$ $=\exp \left[-2 \pi i\left(h, \bar{n}_{v}\right)\right]$.

If we apply the transformation to $Z$, we obtain the right-hand side of (3.1) without the factor of $a^{2}$ and with $h$ replaced by zero. Thus

$$
\langle G(x, y)\rangle=a^{2} Z(h) / Z(0),
$$

with

$$
\begin{aligned}
Z(h)= & \sum_{v: d v=0} \int d \mu(A) \exp \left[-\frac{1}{2}\left(d A+\frac{2 \pi}{e} v, d A+\frac{2 \pi}{e} v\right)\right] \\
& \cdot \exp \left[-i e(A, h)-2 \pi i\left(h, n_{v}\right)\right] .
\end{aligned}
$$

The measure $d \mu(A)$ is the product measure

$$
d \mu(A)=\bar{N}^{-1} \prod_{b \in A^{*}}\left\{\exp \left[a^{2} \cos (e A(b))\right] d A(b)\right\},
$$


where $A(b) \in(-\pi / e, \pi / e)$, and $\bar{N}$ is chosen so that $d \mu(A)$ is a probability measure. We will use this compact version of the model for the remainder of the paper.

In our expansion we will think of the geometrical objects involved as sets of sites rather than sets of bonds and plaquettes. This is a slightly different approach from that of [18]. It has the advantage that the natural definition of connectedness is sufficient to insure that the constraint $d v=0$ factors. We would like to identify a set of bonds or plaquettes with the set of sites belonging to the bonds or plaquettes. However, two different sets of bonds or plaquettes can give rise to the same set of sites. So we make the following definitions.

Definition 3.1. The support of a set of bonds $Z \subset A^{*}$ is

$$
\operatorname{supp} Z=\{x \in \Lambda: x \in b \text { for some bond } b \in Z\} .
$$

The support of a 1-form $f$ is

$$
\operatorname{supp} f=\{x \in \Lambda: x \in b \text { for some bond } b \text { with } f(b) \neq 0\} .
$$

For a set of plaquettes $Y \subset \Lambda^{* *}$ and a 2-form $g$, supp $Y \subset \Lambda$ and $\operatorname{supp} g \subset \Lambda$ are defined similarly. A set $X \subset A$ is connected if any two sites in $X$ can be connected by a path of bonds whose endpoints all lie in $X$. Finally for a connected set $X \subset \Lambda$, $B(X)$ will denote the smallest rectangular parallelepiped in $\Lambda$ which contains $X$.

The individual terms in our expansion will not be independent of the choice of the $n_{v}$ 's. The convergence of the expansion will require further properties for the $n_{v}$ 's. We state these properties here and then show in Appendix B that there is a choice of $n_{v}$ 's having these properties.

Lemma 3.2. For each closed integer-valued 2-form $v$ in three or more dimensions, there is an integer-valued 1-form $n_{v}$ such that

(a) $d n_{v}=v$,

(b) $n_{v}=\sum_{i} n_{v_{t}}$ where $v=\sum_{i} v_{i}$ and $\operatorname{supp} v_{i}$ are the connected components of $\operatorname{supp} v$,

(c) $n_{-v}=-n_{v}$,

(d) $\operatorname{supp} n_{v} \subset B(\operatorname{supp} v)$

(e) $\left\|n_{v}\right\|_{\infty} \leqq c(v, v)^{2}$, where $c$ is a constant which depends only on the number of dimensions.

Remark. We have shown that $Z(h)$ is independent of the choice of the $n_{v}$ 's. The same argument shows $\exp \left[-2 \pi i\left(h, n_{-v}\right)\right]=\exp \left[2 \pi i\left(h, n_{v}\right)\right]$. So by making the change of variables $A \rightarrow-A, v \rightarrow-v$, we see that $Z(-h)=Z(h)$. Equation (3.3) can be used to define $Z(f)$ for any 1-form $f$. However, $Z(f)$ will in general depend on the choice of the $n_{v}$ 's, and the equality $\exp \left[-2 \pi i\left(f, n_{-v}\right)\right]=\exp \left[2 \pi i\left(f, n_{v}\right)\right]$ is no longer automatic. But property (c) of Lemma 3.2 implies this equality, and so $Z(-f)=Z(f)$ for any 1 -form $f$. This property will be important since we will expand about $h=0$.

Our expansion is a standard polymer expansion about $v=0$ and the product measure $d \mu(A)$. (See [18] for a discussion of similar expansions.) For this expansion to converge we will need not only that $e$ is small but also that the mass ae is large compared to the lattice spacing of one. As we saw in Sect. 2, correlation inequalities allow us to enlarge the region of parameters for which we know there is 
LRO. The more sophisticated expansion of [1] is about a Gaussian measure for $A$, and so has a larger domain of convergence. Presumably, this expansion could be adapted to our situation to further enlarge the region of parameters for which we know there is LRO.

For each plaquette $p$ and bond $b$, define $\varrho(p)$ and $\sigma(b)$ by

$$
\begin{aligned}
& 1+\varrho(p)=\exp \left[-\frac{1}{2}|d A(p)|^{2}-\frac{2 \pi}{e} d A(p) v(p)\right], \\
& 1+\sigma(b)=\exp (-i e h(b) A(b)) .
\end{aligned}
$$

Next we expand the products:

$$
\begin{gathered}
\prod_{p \in \Lambda^{* *}}[1+\varrho(p)]=\sum_{Y \subset \Lambda^{* *}} \prod_{p \in Y} \varrho(p), \\
\prod_{b \in \Lambda^{*}}[1+\sigma(b)]=\sum_{Z \subset \Lambda^{*}} \prod_{b \in Z} \sigma(b) .
\end{gathered}
$$

$Y$ and $Z$ are summed over all possible sets of plaquettes and bonds respectively. We now have

$$
Z(h)=\sum_{v: d v=0} \sum_{Y \subset \Lambda^{* *}} \sum_{Z \subset \Lambda^{*}} k(v, Y, Z)
$$

with

$$
k(v, Y, Z)=\exp \left[-\frac{1}{2}\left(\frac{2 \pi}{e}\right)^{2}(v, v)-2 \pi i\left(h, n_{v}\right)\right] \int d \mu(A) \prod_{p \in Y} \varrho(p) \prod_{b \in Z} \sigma(b) .
$$

Given $v, Y$, and $Z$, let $X_{1}, \ldots, X_{n} \subset A$ be the connected components of supp $v$ usupp $Y \cup \operatorname{supp} Z$. We are using the notation of Definition 3.1. Let

$$
\begin{aligned}
Y_{i} & =\left\{p \in Y: p \subset X_{i}\right\}, \\
Z_{i} & =\left\{b \in Z: b \subset X_{i}\right\}, \\
v_{i}(p) & = \begin{cases}v(p) & \text { if } p \subset X_{i} \\
0 & \text { otherwise }\end{cases}
\end{aligned}
$$

If $b \in Z$ or $p \in Y$ then there is exactly one $X_{i}$ which contains all the sites in $b$ or $p$. We have denoted this by $b \subset X_{i}$ or $p \subset X_{i}$. The same is true for a plaquette $p$ with $v(p) \neq 0$. Thus $Y=\bigcup_{i} Y_{i}, Z=\bigcup_{i} Z_{i}, v=\sum_{i} v_{i}$, and $\operatorname{supp} v_{i} \cup \operatorname{supp} Y_{i} \cup \operatorname{supp} Z_{i}=X_{i}$. The sets $\operatorname{supp} v_{i}, \operatorname{supp} Y_{i}$, and $\operatorname{supp} Z_{i}$ need not be connected.

It follows from part (b) of Lemma 3.2 and the fact that $d \mu(A)$ is a product measure that

$$
k(v, Y, Z)=\prod_{i=1}^{n} k\left(v_{i}, Y_{i}, Z_{i}\right) .
$$

We claim that the constraint $d v=0$ is equivalent to $d v_{i}=0$ for $i=1, \ldots, n$. Suppose $d v(c)=0$ for a cube $c$. Our definition of connectedness implies that all the plaquettes in $c$ on which $v$ is nonzero will fall into the same $X_{i}$. So $d v_{i}(c)$ will also be zero. For $j \neq i, d v_{j}(c)$ vanishes trivially. 
We now have

$$
Z(h)=\sum_{n=0}^{\infty} \frac{1}{n !} \sum_{X_{1} \ldots X_{n}}^{\prime} \prod_{i=1}^{n} K\left(X_{i}, h\right),
$$

where the primed sum is over connected subsets $X_{1}, \ldots, X_{n}$ of $A$ with the hard core condition that $X_{i} \cup X_{j}$ is not connected for $i \neq j$. (This implies that $X_{i}$ and $X_{j}$ are disjoint and that no site in $X_{i}$ is a nearest neighbor of a site in $X_{j}$.) Also

$$
K(X, h)=\sum_{\substack{v, Y, Z \\ \text { supp } v \cup \operatorname{supp} Y \cup \operatorname{supp} Z=X}} k(v, Y, Z) .
$$

This sum has the usual constraints on $v, Y$, and $Z$, i.e., $d v=0, Y \subset \Lambda^{*}$, and $Z \subset \Lambda^{* *}$.

In Sect. 5 we will prove the following estimates on $K(X, h)$ and its partial derivatives with respect to the variables $h(b)$. We denote the partial derivative with respect to $h(b)$ by the subscript $b$. For example,

$$
K_{b, b^{\prime}}(X, h)=\frac{\partial^{2}}{\partial h(b) \partial h\left(b^{\prime}\right)} K(X, h) .
$$

Lemma 3.3. For any $M>0$ there exist $\varepsilon>0$ and $\mu<\infty$ such that if $e<\varepsilon$ and ae $>\mu$ then

$$
|K(X, h)| \leqq e^{-M|X|} .
$$

Furthermore, the same bound holds for $K_{b}(X, h), K_{b, b^{\prime}}(X, h)$, and $K_{b, b^{\prime}, b^{\prime \prime}}(X, h)$ where $b, b^{\prime}, b^{\prime \prime} \in \Lambda^{*}$ and $h$ is any 1-form with $\|h\|_{\infty} \leqq c$.

The estimates in Lemma 3.3 are uniform in $\Lambda$. We will use Lemma 3.3 to prove Theorem 2.1 (i). We carry out this proof in a finite volume $\Lambda$, but we will not bother to mention obvious constraints such as $X_{i} \subset \Lambda$. The estimates in the proof will be uniform in $\Lambda$ and so carry over to the infinite volume limit. We prove the existence of the infinite volume limit in the next section. The bound on $K_{b, b^{\prime}, b^{\prime \prime}}(X, h)$ will not be needed until we study the decay of the truncated two point function in the next section.

Proof of Theorem 2.1(i). Our goal is to show that $\langle G(x, y)\rangle$ is close to $a^{2}$ uniformly in $x$ and $y$. By (3.2) this will follow from showing that $\ln Z(h)-\ln Z(0)$ is small uniformly in $x$ and $y$. We will use polymer expansions for $\ln Z(h)$ and $\ln Z(0)$. As noted in Sect. 2, $\|h\|_{2}$ is bounded uniformly in $x$ and $y$ if $d \geqq 3$. This will be the key ingredient in bounding the difference of the two polymer series.

If we choose $M$ large enough in Lemma 3.3, then we have a convergent polymer expansion for $\ln Z(h)$. (For example, see $[5,18]$.)

$$
\ln Z(h)=\sum_{n=1}^{\infty} \frac{1}{n !} \sum_{X_{1}, \ldots, X_{n}} \psi_{c}\left(X_{1}, \ldots, X_{n}\right) \prod_{i=1}^{n} K\left(X_{i}, h\right),
$$

where $\psi_{c}\left(X_{1}, \ldots, X_{n}\right)$ is the connected part of the hard core interaction $\left(X_{i} \cup X_{j}\right.$ is disconnected) in (3.8). The sum is over all choices of connected sets $X_{i}$, but $\psi_{c}\left(X_{1}, \ldots, X_{n}\right)$ vanishes unless $\bigcup_{i} X_{i}$ is connected.

For $s \in[0,1]$ define an interpolating function

$$
H(s)=\ln Z(s h) \text {. }
$$


By the remark following Lemma 3.3, $H(-s)=H(s)$. So $H^{\prime}(0)=0$. Thus

$$
\ln Z(h)-\ln Z(0)=H(1)-H(0)=\int_{0}^{1} d s(1-s) H^{\prime \prime}(s) .
$$

By part (d) of Lemma 3.2, $K(X, h)$ only depends on $h(b)$ with $b \subset B(X)$. Thus

with

$$
H^{\prime \prime}(s)=\sum_{b, b^{\prime}} h(b) h\left(b^{\prime}\right) m\left(b, b^{\prime}\right)
$$

$$
\begin{aligned}
m\left(b, b^{\prime}\right)= & \sum_{n=1}^{\infty} \frac{1}{n !} \sum_{i, j=1}^{n} \sum_{\substack{X_{1}, \ldots, X_{n} \\
b \subset B\left(X_{i}\right), b^{\prime} \subset B\left(X_{j}\right)}} \psi_{c}\left(X_{1}, \ldots, X_{n}\right) \\
& \cdot\left[\prod_{\substack{k=1 \\
k \neq i, j}}^{n} K\left(X_{k}, s h\right)\right] K_{b}\left(X_{i}, s h\right) K_{b^{\prime}}\left(X_{j}, s h\right) .
\end{aligned}
$$

Of course the $i=j$ terms contain $K_{b, b^{\prime}}\left(X_{i}, s h\right)$, not $K_{b}\left(X_{i}, s h\right) K_{b^{\prime}}\left(X_{i}, s h\right)$.

Lemma 3.3 and standard techniques $[5,18]$ can be used to bound $m\left(b, b^{\prime}\right)$. We sketch the argument. The function $\psi_{c}\left(X_{1}, \ldots, X_{n}\right)$ is non-zero only if $\bigcup_{i} X_{i}$ is connected. Thus we can use part of the smallness provided by Lemma 3.3, say $\exp \left(-\frac{1}{2} M|X|\right)$, to extract a factor of $\exp \left[-\frac{1}{2} M d\left(b, b^{\prime}\right)\right]$ from $m\left(b, b^{\prime}\right)$. This leaves us with

$$
\sum_{n=1}^{\infty} \frac{1}{n !} \sum_{i, j=1}^{n} \sum_{\substack{X_{1}, \ldots, X_{n} \\ b \subset \boldsymbol{B}\left(X_{i}\right), \boldsymbol{b}^{\prime} \subset \boldsymbol{B}\left(X_{j}\right)}} \psi_{c}\left(X_{1}, \ldots, X_{n}\right) \exp \left[-\frac{1}{2} M \sum_{i}\left|X_{i}\right|\right] .
$$

We can now drop the constraint $b^{\prime} \subset B\left(X_{j}\right)$. For a fixed $X_{i}$, the sum over the other $X$ 's can be bounded by $\left|X_{i}\right| \delta^{n}(n-1)$ !, where $\delta$ can be made arbitrarily small by making $M$ sufficiently large. Thus we are left with

$$
\sum_{n=1}^{\infty} n \delta^{n} \sum_{X: b \subset B(X)}|X| \exp \left(-\frac{1}{2} M|X|\right) .
$$

The final result is the bound

$$
\left|m\left(b, b^{\prime}\right)\right| \leqq \delta^{\prime} \exp \left[-\frac{1}{2} M d\left(b, b^{\prime}\right)\right]
$$

where $\delta^{\prime}$ can be made arbitrarily small by making $M$ sufficiently large.

By the Cauchy-Schwarz inequality

$$
\left|H^{\prime \prime}(s)\right| \leqq\|h\|_{2}^{2} \sup _{b^{\prime}} \sum_{b}\left|m\left(b, b^{\prime}\right)\right| \leqq \delta^{\prime}\|h\|_{2}^{2} \sum_{b} \exp \left[-\frac{1}{2} M d\left(b, b^{\prime}\right)\right] .
$$

For $d \geqq 3,\|h\|_{2}^{2}$ is finite and bounded uniformly in $x, y$ and $\Lambda$. Combining (3.12) and (3.16) completes the proof.

\section{Decay of the Truncated Correlation}

In this section we will prove Theorem 2.5 , which establishes massless decay for the truncated correlation $\langle G(x, y)\rangle-G_{\infty}$. In order to do this, we first take the infinite volume limit of $\langle G(x, y)\rangle$. 
Up till now we have considered the model only in a finite volume. In this section we will distinguish finite volume quantities with a subscript $\Lambda$. So the expectation of the smeared string (2.5) becomes $\left\langle G_{\Lambda}(x, y)\right\rangle_{\Lambda}$. Note that $G_{\Lambda}(x, y)$ depends on $\Lambda$ because $h_{A}=d_{\Lambda} C_{A} g$ depends on $A$. The existence of the infinite volume limit is usually an immediate consequence of the convergence of the polymer expansion. However, in our case it is also necessary that $h_{A}$ converges in $\ell^{2}$ as $\Lambda \nearrow \mathbb{Z}^{d}$. Let $h$ denote $d C g$, where now $C$ is the inverse of the infinite volume lattice Laplacian. We extend $h_{A}$ to a 1 -form on $\mathbb{Z}^{d}$ by defining $h_{A}$ to be zero outside $\Lambda^{*}$.

Lemma 4.1. In $d \geqq 3, h_{A}$ converges to $h$ in $\ell^{2}$.

Proof. It suffices to show $\left(h_{A}, h\right)$ and $\left(h_{A}, h_{A}\right)$ converge to $(h, h)$. However using $d^{*} h_{\Lambda}=d_{\Lambda}^{*} h_{\Lambda}=g$, we have

$$
\left(h_{\Lambda}, h\right)=\left(h_{\Lambda}, d C g\right)=\left(d^{*} h_{\Lambda}, C g\right)=(g, C g)=(h, h) .
$$

Furthermore $\left(h_{A}, h_{A}\right)=\left(g, C_{A} g\right)$, so the proof reduces to the pointwise convergence of $C_{A}$ to $C$, which is standard.

Lemma 4.2. The infinite volume limit of $\left\langle G_{A}(x, y)\right\rangle_{A}$ exists and equals $a^{2} \exp F(h)$, where

$$
F(h)=\sum_{n=1}^{\infty} \frac{1}{n !} \sum_{X_{1}, \ldots, X_{n}} \psi_{c}\left(X_{1}, \ldots, X_{n}\right)\left[\prod_{i} K\left(X_{i}, h\right)-\prod_{i} K\left(X_{i}, 0\right)\right] .
$$

The $X_{i}$ 's are summed over connected subsets of $\mathbb{Z}^{d}$.

Proof. Since $\left\langle G_{A}(x, y)\right\rangle_{A}=a^{2} \exp \left[\ln Z_{A}\left(h_{A}\right)-\ln Z_{A}(0)\right]$, it is sufficient to show $F(h)-F\left(h_{\Lambda}\right)$ and $F\left(h_{\Lambda}\right)-\ln Z_{\Lambda}\left(h_{A}\right)+\ln Z_{\Lambda}(0)$ both converge to zero as $\Lambda \rtimes \mathbb{Z}^{d}$. To bound the first expression we use the interpolating function

$$
H(s, t)=F\left(\frac{1}{2} s\left(h+h_{A}\right)+\frac{1}{2} t\left(h-h_{A}\right)\right)-F\left(\frac{1}{2} s\left(h+h_{A}\right)-\frac{1}{2} t\left(h-h_{A}\right)\right) .
$$

Since $F(h)=F(-h)$,

$$
F(h)-F\left(h_{\Lambda}\right)=\int_{0}^{1} d t \int_{0}^{1} d s \frac{\partial^{2} H}{\partial s \partial t} .
$$

As in the proof of Theorem 2.1 (i) in Sect. 3,

$$
\frac{\partial^{2} H}{\partial s \partial t}=\sum_{b, b^{\prime}} m\left(b, b^{\prime}\right)\left(h+h_{A}\right)(b)\left(h-h_{A}\right)\left(b^{\prime}\right),
$$

which is bounded in absolute value by $c\left\|h+h_{A}\right\|_{2}\left\|h-h_{A}\right\|_{2}$, since

$$
\left|m\left(b, b^{\prime}\right)\right| \leqq e^{-M^{\prime} d\left(b, b^{\prime}\right)} .
$$

So Lemma 4.1 implies $F(h)-F\left(h_{A}\right)$ converges to zero.

The term $F\left(h_{A}\right)-\ln Z_{A}\left(h_{A}\right)+\ln Z_{A}(0)$ equals expression (4.1) with the constraint that $\bigcup_{i} X_{i}$ contains sites in both $\Lambda$ and $\mathbb{Z}^{d} \backslash \Lambda$. Let $H(s)$ be this expression with $h_{\Lambda}$ replaced by $s h_{\Lambda}$. Again following the proof of Theorem 2.1 (i) in Sect. 3,

$$
F\left(h_{\Lambda}\right)-\ln Z_{\Lambda}\left(h_{\Lambda}\right)+\ln Z_{\Lambda}(0)=\int_{0}^{1} d s(1-s) H^{\prime \prime}(s)
$$


and

with

$$
H^{\prime \prime}(s)=\sum_{b, b^{\prime}} m\left(b, b^{\prime}\right) h_{\Lambda}(b) h_{\Lambda}\left(b^{\prime}\right)
$$

$$
\left|m\left(b, b^{\prime}\right)\right| \leqq e^{-M^{\prime}\left[d(b, \partial \Lambda)+d\left(b^{\prime}, \partial \Lambda\right)+d\left(b, b^{\prime}\right)\right]} .
$$

This bound on $m\left(b, b^{\prime}\right)$ and the convergence of $h_{\Lambda}$ to $h$ in $\ell^{2}$ imply that $F\left(h_{A}\right)$ $-\ln Z_{\Lambda}\left(h_{\Lambda}\right)+\ln Z_{\Lambda}(0)$ converges to zero.

One expects the limit of $F(h)$ as $|x-y| \rightarrow \infty$ to be $2 F\left(h_{0}\right)$, where $h_{0}=d C \delta_{0}$ with $\delta_{0}$ the delta function at the origin. So we consider

$$
\langle G(x, y)\rangle-a^{2} e^{2 F\left(h_{0}\right)}=a^{2} e^{2 F\left(h_{0}\right)}\left[e^{F(h)-2 F\left(h_{0}\right)}-1\right] .
$$

We will show that $F(h)-2 F\left(h_{0}\right)$ equals $\sigma|x-y|^{2-d}$ to leading order. This will prove Theorem 2.5 and will also show that

$$
G_{\infty}=a^{2} e^{2 F\left(h_{0}\right)} \text {. }
$$

Therefore the proof of Theorem 2.5 is reduced to the following proposition.

\section{Proposition 4.3.}

$$
F(h)-2 F\left(h_{0}\right)=\frac{\sigma}{|x-y|^{d-2}}+O\left(\frac{1}{|x-y|^{d-1}}\right) .
$$

Proof. We use Taylor's theorem to extract the leading order term in $F(h)-2 F\left(h_{0}\right)$. We can write $h=h^{x}-h^{y}$, where $h^{x}=d C \delta_{x}$. By translation invariance, $F\left(h_{0}\right)=F\left(h^{z}\right)$ for all $z$. So define

Then

$$
H(s, t)=F\left(s h^{x}-t h^{y}\right)-F\left(s h^{x}\right)-F\left(t h^{y}\right) .
$$

$$
\begin{aligned}
F(h)-2 F\left(h_{0}\right) & =H(1,1)=\int_{0}^{1} \int_{0}^{1} d s d t \frac{\partial^{2} H}{\partial s \partial t}(s, t) \\
& =\frac{\partial^{2} H}{\partial s \partial t}(0,0)+\int_{0}^{1} d t(1-t) \frac{\partial^{3} H}{\partial s \partial t^{2}}(0, t)+\int_{0}^{1} \int_{0}^{1} d s d t(1-s) \frac{\partial^{3} H}{\partial s^{2} \partial t}(s, t) .
\end{aligned}
$$

The leading order term $\sigma|x-y|^{2-d}$ comes from $\frac{\partial^{2} H}{\partial s \partial t}(0,0)$. The $O\left(|x-y|^{1-d}\right)$ terms come partly from $\frac{\partial^{2} H}{\partial s \partial t}(0,0)$ and partly from the higher derivatives in (4.6). These higher derivatives can be easily computed. They give

$$
\frac{\partial^{3} H}{\partial s \partial t^{2}}=\sum_{b, b^{\prime}, b^{\prime \prime}} h^{x}(b) h^{y}\left(b^{\prime}\right) h^{y}\left(b^{\prime \prime}\right) q\left(b, b^{\prime}, b^{\prime \prime}\right) .
$$

The same argument used in the proof of Theorem 2.1 (i) in Sect. 3 gives the bound

$$
\left|q\left(b, b^{\prime}, b^{\prime \prime}\right)\right| \leqq \delta^{\prime} \exp \left[-\frac{1}{2} M d\left(b, b^{\prime}, b^{\prime \prime}\right)\right],
$$

where $d\left(b, b^{\prime}, b^{\prime \prime}\right)$ is the size of the smallest connected set of sites containing $b, b^{\prime}$, and $b^{\prime \prime}$. As $|x-y| \rightarrow \infty, \sum_{b} h^{x}(b) h^{y}(b)^{2}$ behaves asymptotically like $|x-y|^{1-d}$. We leave it to the reader to use (4.8) to show that (4.7) has the same asymptotic behavior. 
The leading order term in (4.6) can be written

with

$$
\frac{\partial^{2} H}{\partial s \partial t}(0,0)=\sum_{b, b^{\prime}} h^{x}(b) h^{y}\left(b^{\prime}\right) m\left(b, b^{\prime}\right)
$$

$$
m\left(b, b^{\prime}\right)=-\sum_{n=1}^{\infty} \frac{1}{n !} \sum_{i=1}^{n} \sum_{\substack{X_{1}, \ldots, X_{n}: \\ b, b^{\prime} \in \boldsymbol{B}\left(X_{\imath}\right)}} \psi_{c}\left(X_{1}, \ldots, X_{n}\right)\left[\prod_{j \neq i} K\left(X_{j}, 0\right)\right] K_{b, b^{\prime}}\left(X_{i}, 0\right),
$$

where we have used $K_{b}(X, 0)=0$.

We can separate (4.9) into two sums. The first sum is over bonds $b$ and $b^{\prime}$ which are parallel. This sum can be written as

$$
\frac{1}{2} \sum_{z} \sum_{b}\left[h^{x}\left(T_{z} b\right) h^{y}(b)+h^{x}(b) h^{y}\left(T_{z} b\right)\right] m\left(b, T_{z} b\right),
$$

where $T_{z} b$ is the translate of the bond $b$ by the lattice vector $z$. We have used the fact that $m\left(b, b^{\prime}\right)=m\left(b^{\prime}, b\right)$. This expression equals

$$
\sum_{z, b} h^{x}(b) h^{y}(b) m\left(b, T_{z} b\right)-\frac{1}{2} \sum_{z, b} m\left(b, T_{z} b\right)\left[h^{x}\left(T_{z} b\right)-h^{x}(b)\right]\left[h^{y}\left(T_{z} b\right)-h^{y}(b)\right] .
$$

We define

$$
m_{0}=\sum_{z} m\left(b, T_{z} b\right) \text {. }
$$

This is independent of $b$, so the first sum in (4.12) is

$$
m_{0}\left(h^{x}, h^{y}\right)=m_{0} C(x, y)=\frac{\sigma}{|x-y|^{d-2}}+O\left(\frac{1}{|x-y|^{d-1}}\right) .
$$

The expression (4.10) implies that $m\left(b, T_{z} b\right)$ decays exponentially in $|z|$. The leading order contribution to $m_{0}$ comes from the $z=0$ term in (4.13), and this is positive for ae sufficiently large and $e$ sufficiently small. We can use bounds like

$$
\left|h^{x}\left(T_{z} b\right)-h^{x}(b)\right| \leqq c|z| \operatorname{dist}(x, b)^{-d}
$$

and the exponential decay of $m\left(b, T_{z} b\right)$ in $|z|$ to show that the second sum in (4.12) is $O\left(|x-y|^{1-d}\right)$.

The second sum in (4.9) is over $b$ and $b^{\prime}$ which are not parallel, i.e., $b$ and $b^{\prime}$ point in orthogonal directions. Let $R_{b} b^{\prime}$ be the reflection of $b^{\prime}$ in the line through $b$. Then $m\left(b, b^{\prime}\right)=m\left(b, R_{b} b^{\prime}\right)$, so we can write the second sum as

$$
\frac{1}{4} \sum_{b, b^{\prime}: \perp} m\left(b, b^{\prime}\right)\left[h^{x}(b)+h^{x}\left(R_{b^{\prime}} b\right)\right]\left[h^{y}\left(b^{\prime}\right)+h^{y}\left(R_{b} b^{\prime}\right)\right] .
$$

Note that $R_{b}$ changes the orientation of bonds which are not parallel to $b$. For each $b$ and $b^{\prime}$ there is a lattice vector $z$ such that $R_{b}, b$ is $T_{z} b$ with its orientation reversed. So

$$
h^{x}(b)+h^{x}\left(R_{b^{\prime}} b\right)=h^{x}(b)-h^{x}\left(T_{z} b\right) .
$$

The length of $z$ is bounded by $2 d\left(b, b^{\prime}\right)$. Thus (4.14) can be bounded in the same way as the second sum in (4.12) and so is $O\left(|x-y|^{1-d}\right)$. 


\section{Bounds on $K(X, h)$}

We now complete the proofs in Sects. 3 and 4 by proving the bounds on $K(X, h)$ and its derivatives stated in Lemma 3.3. We will prove these bounds for $e$ small and ae large. We must show that there is a small factor $e^{-M}$ for each site in $X$. This smallness arises in two different ways. For plaquettes $p$ with $v(p) \neq 0$, the factor $\exp \left[-\frac{1}{2}\left(\frac{2 \pi}{e}\right)^{2} v(p)^{2}\right]$ is small since $e$ is small. For plaquettes $p \in Y$ with $v(p)=0$, the factor $\varrho(p)$ provides smallness because $A$ has a large mass $a e$. For bonds $b \in Z$, the factor $\sigma(b)$ provides smallness for the same reason.

We will first bound $K(X, h)$ and then show why the same bounds hold for the derivatives $K_{b}(X, h), K_{b, b^{\prime}}(X, h)$, and $K_{b, b^{\prime}, b^{\prime \prime}}(X, h)$. Recall that $K(X, h)$ is a sum of terms $k(v, Y, Z)$ with $X=\operatorname{supp} v \cup \operatorname{supp} Y \cup \operatorname{supp} Z$ [see Eq. (3.9)]. For a plaquette $p \in Y$ with $v(p) \neq 0$, we use

$$
\frac{2 \pi}{e} d A(p) v(p) \leqq d A(p)^{2}+\frac{1}{4}\left(\frac{2 \pi}{e}\right)^{2} v(p)^{2} .
$$

So

$$
\begin{aligned}
\varrho \varrho(p) \mid & =\left|\exp \left[-\frac{1}{2} d A(p)^{2}-\frac{2 \pi}{e} d A(p) v(p)\right]-1\right| \\
& \leqq 2 \exp \left[\frac{1}{2} d A(p)^{2}+\frac{1}{4}\left(\frac{2 \pi}{e}\right)^{2} v(p)^{2}\right] .
\end{aligned}
$$

For a plaquette $p \in Y$ with $v(p)=0$,

$$
\varrho \varrho(p)|=| e^{\frac{-1}{2} d A(p)^{2}}-1 \mid \leqq \frac{1}{2} d A(p)^{2} .
$$

Finally for a bond $b \in Z$, we use

$$
|\sigma(b)|=\left|e^{-i e A(b) h(b)}-1\right| \leqq e|A(b) h(b)| \leqq c_{1} e|A(b)|,
$$

since $\|h\|_{\infty}$ is bounded uniformly in $x, y$, and $\Lambda$.

Combining these bounds gives

$$
\begin{aligned}
|k(v, Y, Z)| \leqq & \exp \left[-\frac{1}{4}\left(\frac{2 \pi}{e}\right)^{2}(v, v)\right] \int d \mu(A) \prod_{\substack{p \in Y: \\
v(p) \neq 0}} 2 \exp \left[\frac{1}{2} d A(p)^{2}\right] \\
& \cdot \prod_{\substack{p \in Y: \\
v(p)=0}} \frac{1}{2} d A(p)^{2} \prod_{b \in Z} c_{1} e|A(b)| .
\end{aligned}
$$

By the Cauchy-Schwarz inequality,

Thus

$$
d A(p)^{2} \leqq 4 \sum_{b \in p} A(b)^{2} .
$$

$$
\prod_{\substack{p \in Y \\ v(p) \neq 0}} \exp \left[\frac{1}{2} d A(p)^{2}\right] \leqq \exp \left[2 r \sum_{b \subset X} A(b)^{2}\right],
$$

where $r=2(d-1)$ is the number of plaquettes which contain a fixed bond. 
Therefore (5.1) is bounded by a sum of terms of the form

$$
c_{2}^{|X|} \exp \left[-\ln e^{-1}|Z|-\frac{1}{4}\left(\frac{2 \pi}{e}\right)^{2}(v, v)^{2}\right] \int d \mu(A) \exp \left[2 r \sum_{b \subset X} A(b)^{2}\right] \prod_{b \subset X}|A(b)|^{q(b)},
$$

with $q(b) \leqq 2 r+1$ and $\sum_{b \subset X} q(b)=2|Y \backslash v|+|Z|$. We have denoted $\{p \in Y: v(p)=0\}$ by $Y \backslash v ;|Y \backslash v|$ is the number of plaquettes in $Y \backslash v$, and $|Z|$ is the number of bonds in $Z$. The number of terms of the form (5.2) is bounded by $4^{|Y \backslash v|}$. (Note: the " $e$ " in $\ln e^{-1}$ is the electric charge.)

Lemma 5.1. For any $M^{\prime}>0, \exists \mu>0$ such that for ae $>\mu$,

$$
\int d \mu(A) e^{2 r A(b)^{2}}|A(b)|^{q(b)} \leqq c_{3} e^{-M^{\prime} q(b)} .
$$

Proof. Since $d \mu(A)$ is a product measure over bonds, (5.3) reduces to the onedimensional integral

$$
\frac{1}{N} \int_{-\pi / e}^{\pi / e} d A \exp \left\{a^{2}[\cos (e A)-1]+2 r A^{2}\right\}|A|^{q(b)},
$$

where

$$
N=\int_{-\pi / e}^{\pi / e} d A \exp \left\{a^{2}[\cos (e A)-1]\right\}
$$

By rescaling $A$ by $\frac{1}{e}$ and using simple bounds, we get (5.3) with $M^{\prime}=\ln (a e)$ for ae sufficiently large.

Since $d \mu(A)$ is a product measure, Lemma 5.1 gives the bound

$$
\begin{aligned}
(5.2) & \leqq c_{4}^{|X|} \exp \left[-\frac{1}{4}\left(\frac{2 \pi}{e}\right)^{2}(v, v)-2 M^{\prime}|Y \backslash v|-\left(M^{\prime}+\ln e^{-1}\right)|Z|\right] \\
& \leqq c_{4}^{|X|} e^{-M^{\prime \prime}|X|} \exp \left[-\frac{1}{8}\left(\frac{2 \pi}{e}\right)^{2}(v, v)\right],
\end{aligned}
$$

where $M^{\prime \prime}$ can be made as large as desired by taking $M^{\prime}$ large enough and $e$ small enough. We have used $X=\operatorname{supp} v \cup \operatorname{supp} Y \cup \operatorname{supp} Z$ to bound $|X|$ by $4(v, v)+4|Y \backslash v|$ $+2|Z|$. The number of choices of $Y$ and $Z$ is bounded by $c^{|X|}$, as is the number of terms of the form (5.2). So

$$
\begin{aligned}
|K(X, h)| & =\left|\sum_{v, Y, Z} k(v, Y, Z)\right| \\
& \leqq c_{5}^{|X|} e^{-M^{\prime \prime}|X|} \sum_{v} \exp \left[-\frac{1}{8}\left(\frac{2 \pi}{e}\right)^{2}(v, v)\right] \\
& \leqq c_{6}^{|X|} e^{-M^{\prime \prime}|X|} .
\end{aligned}
$$

This proves part (a) of Lemma 3.3 since $M^{\prime \prime}$ can be made arbitrarily large by taking $e$ small and ae large.

The derivatives of $k(v, Y, Z)$ with respect to $h(b)$ can either pull down a factor of $2 \pi i n_{v}(b)$, or change $\sigma(b)$ to $i e A(b) e^{-i e A(b) h(b)}$. Additional derivatives produce similar factors. By Lemma 3.2 part (e), $\left|n_{v}(b)\right| \leqq c(v, v)^{2}$, and so the sum over $v$ in (5.4) may 
contain an additional factor $(v, v)^{2 p}$, where $p=1,2$ or 3 . However, the sum is still bounded by $c^{|X|}$. The extra factors of $e A(b)$ coming from the derivatives of $\sigma(b)$ may increase $\sum_{b \subset X} q(b)$, but this only improves our estimates.

\section{Appendix A}

In this appendix we will prove a correlation inequality which relates expectations in the fixed length and variable length models. The proof is quite similar to the proofs of correlation inequalities presented in [6]. The analog of this inequality for a one-component classical spin system was proved by Wells [19]. Bricmont et al. [4] remarked that the inequality also holds for a two-component classical spin system. We first introduce duplicate variables and consider the integral

$$
\begin{aligned}
I= & \int d \mu_{c}(A) d \mu_{c}\left(A^{\prime}\right) d v(r) D \theta D \theta^{\prime} \\
& \cdot\left\{r(x) r(y) \cos [(\theta, g)-e(A, h)]-a^{2} \cos \left[\left(\theta^{\prime}, g\right)-e\left(A^{\prime}, h\right)\right]\right\} \\
& \cdot \exp \left[\sum_{\langle z w\rangle} r(z) r(w) \cos (d \theta-e A)+a^{2} \sum_{\langle z w\rangle} \cos \left(d \theta^{\prime}-e A^{\prime}\right)\right] .
\end{aligned}
$$

The measure $d v(r)$ is the product over sites of the radial measure $r d r \exp \left[-\lambda\left(r^{2}-\sigma^{2}\right)^{2}\right]$, where

$$
\sigma^{2}=\frac{1}{2 \lambda}\left(-\frac{1}{2} m^{2}-d\right) .
$$

Also $d \mu_{c}(A)$ is the gaussian measure with covariance

$$
C=\left(d^{*} d+\frac{1}{\alpha} d d^{*}\right)^{-1}
$$

and the sums are over bonds $\langle z w\rangle$ in $\Lambda^{*}$. Since we wish to prove that $\langle G(x, y)\rangle^{\prime}$ $\geqq\langle G(x, y)\rangle$, it is sufficient to prove that $I \geqq 0$. We will prove that $I \geqq 0$ provided

$$
2 a \leqq \sigma .
$$

This implies Theorem 2.3, since by taking $e$ sufficiently small and $a$ sufficiently large, we have $\langle G(x, y)\rangle \geqq c$. Then (A.1) implies the same for $\langle G(x, y)\rangle^{\prime}$ for any $\lambda$ provided $-m^{2}$ is sufficiently large.

We wish to make the following change of variables; define

$$
\alpha=A^{\prime}-A, \quad \beta=A^{\prime}+A, \quad \psi=\frac{1}{2}\left(\theta^{\prime}+\theta\right), \quad \phi=\frac{1}{2}\left(\theta^{\prime}-\theta\right) .
$$

In order to see how the terms in $I$ change as a result we will use the following identity:

$$
\begin{aligned}
4\left(x y z-x^{\prime} y^{\prime} z^{\prime}\right)= & \left(x+x^{\prime}\right)\left(y+y^{\prime}\right)\left(z-z^{\prime}\right)+\left(x-x^{\prime}\right)\left(y-y^{\prime}\right)\left(z-z^{\prime}\right) \\
& +\left(x+x^{\prime}\right)\left(y-y^{\prime}\right)\left(z+z^{\prime}\right)+\left(x-x^{\prime}\right)\left(y+y^{\prime}\right)\left(z+z^{\prime}\right) .
\end{aligned}
$$

There is a corresponding identity for $x y z+x^{\prime} y^{\prime} z^{\prime}$. We will apply these identities to the terms in $I$ taking $x$ and $y$ equal to $r(\cdot), x^{\prime}$ and $y^{\prime}$ equal to $a$, and $z$ and $z^{\prime}$ equal to the cosines of unprimed and primed variables. Expanding out the exponential in a 
power series, we get a sum of terms with positive coefficients. It is sufficient to show that a typical term is positive. Using simple trigonometric identities a typical term can be written

$$
\int D v(r) \prod_{z}(r(z)-a)^{n(z)}(r(z)+a)^{m(z)}
$$

$$
\left[\int d \mu_{\bar{c}}(\alpha) D \psi \prod_{\langle z w\rangle}\left\{\cos ^{n(z, w)}\left(d \psi-\frac{e}{2} \alpha\right) \sin ^{m(z, w)}\left(d \psi-\frac{e}{2} \alpha\right)\right\} f\left((\psi, g)-\frac{e}{2}(\alpha, h)\right)\right]^{2},
$$

where $n(z), m(z), n(z, w), m(z, w)$ are non-negative integers, $\bar{C}=2 C$ and $f$ is either $\sin$ or cos. Therefore it remains to prove positivity of the radial integral. Since it factorizes over sites, we just need to prove positivity of the following integral,

$$
K_{n, m}=\int_{0}^{\infty} r d r e^{-\lambda\left(r^{2}-\sigma^{2}\right)^{2}}(r-a)^{n}(r+a)^{m} .
$$

By rescaling, we can set $\sigma=1$. Simple estimates show that

$$
K_{n, m} \geqq(1-a)^{n}(1+a)^{m} \int_{1}^{\infty} r d r e^{-\lambda\left(r^{2}-1\right)^{2}}-a^{n}(2 a)^{m} \int_{0}^{a} r d r e^{-\lambda\left(r^{2}-1\right)^{2}} .
$$

For any $\lambda>0$, we have

$$
\int_{1}^{\infty} r d r e^{-\lambda\left(r^{2}-1\right)^{2}} \geqq \int_{0}^{1} r d r e^{-\lambda\left(r^{2}-1\right)^{2}},
$$

and therefore $K_{n, m} \geqq 0$ for all $n, m$ if $a \leqq \frac{1}{2}$.

\section{Appendix B}

This appendix is devoted to the construction of integer-valued 1-forms $n_{v}$ which have the properties listed in Lemma 3.2. We will first carry out this construction for closed 2-forms $v$ which have connected support. The construction is in two steps. First we construct a 1 -form $n_{v}^{\prime}$ with $d n_{v}^{\prime}=v$. A bound on $\left\|n_{v}^{\prime}\right\|_{\infty}$ is immediate from this construction, but $\operatorname{supp} n_{v}^{\prime}$ need not be contained in $B(\operatorname{supp} v)$. So the second step is to gauge transform $n_{v}^{\prime} \rightarrow n_{v}$ so that $\operatorname{supp} n_{v} \subset B(\operatorname{supp} v)$.

We construct $n_{v}^{\prime}$ using a maximal tree. To avoid technical problems we choose a particular such tree $T \subset \Lambda^{*}$. We choose coordinates $\left(x_{0}, x_{1}, \ldots, x_{d-1}\right)$ for the points in $\Lambda$ so that $x_{i}=0,1, \ldots, L_{i}$. $T$ contains all the bonds in the 0 -direction, all the bonds in the 1-direction which lie in the $x_{0}=0$ hyperplane, all the bonds in the 2-direction which lie in the $x_{0}=0$ and $x_{1}=0$ hyperplanes, $\ldots$, and all the bonds in the $(d-1)$-direction which lie in the $x_{0}=0, x_{1}=0, \ldots$, and $x_{d-2}=0$ hyperplanes. The origin is a natural base for this tree.

For any $x \in \Lambda$ there is a unique path $\Gamma_{x}$ in $T$ from the origin to $x$. Given $\langle x y\rangle \in \Lambda^{*}$, let $S_{x y}$ be a surface whose boundary is the loop $\langle x y\rangle \cup\left(-\Gamma_{y}\right) \cup \Gamma_{x}$. Define

$$
n_{v}^{\prime}(\langle x y\rangle)=\sum_{p \in S_{x y}} v(p)
$$

The surface $S_{x y}$ is not unique. However, $n_{v}^{\prime}$ is well defined because $v$ is closed. We leave it to the reader to check that $d n_{v}^{\prime}=v$, i.e., property (a) holds. Definition B.1 
implies $\left\|n_{v}^{\prime}\right\|_{\infty} \leqq\|v\|_{1} \leqq(v, v)$. The second inequality holds because $v$ is integervalued.

The only thing wrong with $n_{v}^{\prime}$ is that it can have support outside of $B(\operatorname{supp} v)$. We rectify this by defining a 0 -form $s$ so that $n_{v}^{\prime}=d s$ outside $B(\operatorname{supp} v)$ and then letting $n_{v}=n_{v}^{\prime}-d s$. Fix a point $x_{0}$ just outside of $B(\operatorname{supp} v)$ so $x_{0} \notin \operatorname{supp} v$, but some nearest neighbor of $x_{0}$ is in $B(\operatorname{supp} v)$. For $x \in \Lambda \backslash B(\operatorname{supp} v)$ let

$$
s(x)=\sum_{b \in \Gamma_{x_{0}, x}} n_{v}^{\prime}(b),
$$

where $\Gamma_{x_{0}, x}$ is a path from $x_{0}$ to $x$ which is entirely outside of $B(\operatorname{supp} v)$. In three or more dimensions closed paths in $\Lambda \backslash B(\operatorname{supp} v$ ) can be expressed as the boundary of a surface which lies entirely in $\Lambda \backslash B(\operatorname{supp} v)$. But $d n_{v}^{\prime}(p)=v(p)=0$ for plaquettes $p$ in $\Lambda \backslash B(\operatorname{supp} v)$, so $s(x)$ is independent of the path $\Gamma_{x_{0}, x}$ and so is well defined for $x \in \Lambda \backslash B(\operatorname{supp} v)$. Furthermore

$$
d s(b)=n_{v}^{\prime}(b) \text { for } \quad b \subset \Lambda \backslash B(\operatorname{supp} v) .
$$

If $x$ is just inside of $B(\operatorname{supp} v)$ we define $s(x)$ as follows. There is a path $\Gamma_{x_{0}, x}$ from $x_{0}$ to $x$ such that no bond $b$ in $\Gamma_{x_{0}, x}$ lies entirely in $B(\operatorname{supp} v)$. Define $s(x)$ by (B.2). For plaquettes $p$ which contain sites both in $B(\operatorname{supp} v)$ and in $\Lambda \backslash B(\operatorname{supp} v)$, $d n_{v}^{\prime}(p)=0$. So the argument of the preceding paragraph shows $s(x)$ is well defined and $d s(b)=n_{v}^{\prime}(b)$ for bonds $b$ which connect $B(\operatorname{supp} v)$ and $\Lambda \backslash B(\operatorname{supp} v)$.

For the remaining $x$ in $B(\operatorname{supp} v)$, we let $s(x)=0$. Define

$$
n_{v}=n_{v}^{\prime}-d s .
$$

Then $\operatorname{supp} n_{v} \subset B(\operatorname{supp} v)$, i.e., property (d) holds. Property (a) still holds since $d^{2} s=0$. To bound $\left\|n_{v}\right\|_{\infty}$, we need a bound on $d s(b)$ for $b \subset B(\operatorname{supp} v)$. By choosing the path in (B.2) to have minimal length, we get

$$
|s(x)| \leqq\left\|n_{v}^{\prime}\right\|_{\infty} \ell(v),
$$

for $x \in B(\operatorname{supp} v)$, where $\ell(v)$ is the sum of the lengths of the sides of $B(\operatorname{supp} v)$. Hence

$$
\left\|n_{v}\right\|_{\infty} \leqq\left\|n_{v}^{\prime}\right\|_{\infty}+2\left\|n_{v}^{\prime}\right\|_{\infty} \ell(v) .
$$

But supp $v$ is connected, so $\ell(v) \leqq c_{1}(v, v)$ for a constant $c_{1}$ which only depends on the number of dimensions. Hence

$$
\left\|n_{v}\right\|_{\infty} \leqq c(v, v)^{2},
$$

which is property (e).

As noted in Lemma 3.2, any 2-form $v$ can be uniquely written as $\sum_{i} v_{i}$, where $\operatorname{supp} v_{i}$ are the connected components of $\operatorname{supp} v$. As shown in the discussion preceding (3.8), if $d v=0$ then $d v_{i}=0$. So we can define

Then property $(b)$ is satisfied.

$$
n_{v}=\sum_{i} n_{v_{i}} .
$$

Property (c) remains to be verified. The closed 2 -forms $v$ may be grouped into pairs $v$ and $-v$. For each pair we can define $n_{v}$ as above and then define $n_{-v}$ to be $-n_{v}$. 
Acknowledgements. It is a pleasure to thank C. Borgs, E. Lieb, F. Nill, E. Seiler, and A. Wightman for valuable discussions.

\section{References}

1. Bałaban, T., Brydges, D., Imbrie, J., Jaffe, A.: The mass gap for Higgs models on a unit lattice. Ann. Phys. 158, 281-319 (1984)

2. Bernstein, J.: Spontaneous symmetry breaking, gauge theories, the Higgs mechanism and all that. Rev. Mod. Phys. 46, 7-48 (1974)

3. Bricmont, J., Fröhlich, J.: An order parameter distinguishing between different phases of lattice gauge theories with matter fields. Phys. Lett. 122 B, 73-77 (1983)

4. Bricmont, J., Lebowitz, J.L., Pfister, C.-E.: Periodic Gibbs states of ferromagnetic spin systems. J. Stat. Phys. 24, 269-277 (1981)

5. Brydges, D.: A short course on cluster expansions. In: Proceedings of 1984 Les Houches summer school. Osterwalder, K. (ed.) (to be published)

6. Brydges, D., Fröhlich, J., Seiler, E.: On the construction of quantized gauge fields. I. General results. Ann. Phys. 121, 227-284 (1979)

7. Brydges, D., Fröhlich, J., Seiler, E.: Diamagnetism and critical properties of Higgs lattice gauge theories. Nucl. Phys. B 152, 521-532 (1979)

8. Brydges, D., Seiler, E.: Absence of screening in certain lattice gauge and plasma models. Princeton University preprint

9. Drühl, K., Wagner, H.: Algebraic formulation of duality transformations for abelian lattice models. Ann. Phys. 141, 225-253 (1982)

10. Fröhlich, J., Morchio, G., Strocchi, F.: Higgs phenomenon without symmetry breaking order parameter. Nucl. Phys. B 190, 553-582 (1981)

11. Fröhlich, J., Simon, B., Spencer, T.: Infrared bounds, phase transitions and continuous symmetry breaking. Commun. Math. Phys. 50, 79-95 (1976)

12. Fröhlich, J., Spencer, T.: Massless phases and symmetry restoration in abelian gauge theories and spin systems. Commun. Math. Phys. 83, 411-454 (1982)

13. Fredenhagen, K., Marcu, M.: Charged states in $\mathbb{Z}_{2}$ gauge theories. Commun. Math. Phys. 92 , 81-119 (1983)

Fredenhagen, K., Marcu, M.: A confinement criterion for QCD with dynamical quarks, DESY preprint 85-008

14. Guth, A.: Existence proof of a nonconfining phase in four dimensional U(1) lattice gauge theory. Phys. Rev. D 21, 2291-2307 (1980)

15. Kennedy, T., King, C.: Symmetry breaking in the lattice abelian Higgs model. Phys. Rev. Lett. 55, 776-778 (1985)

16. McBryan, O., Spencer, T.: On the decay of correlations in $\mathrm{SO}(n)$-symmetric ferromagnets. Commun. Math. Phys. 53, 299-302 (1977)

17. Osterwalder, K., Seiler, E.: Gauge field theories on a lattice. Ann. Phys. 110, 440-471 (1978)

18. Seiler, E.: Gauge theories as a problem of constructive quantum field theory and statistical mechanics. Lecture Notes in Physics, Vol. 159. Berlin, Heidelberg, New York: Springer 1982

19. Wells, D.: Thesis, Indiana University (1977)

Communicated by A. Jaffe

Received September 19, 1985; in revised form November 15, 1985 
